\title{
La intervención del trabajador social desde las redes sociales en trabajo social comunitario: fundamentos, dimensiones y competencias
}

\author{
María Dolores Rodríguez Álvarez \\ Ayuntamiento de Getafe (Madrid) \\ <lola-rodriguez@hotmail.es>
}

Gizarte-langileek berauen jarduera profesionalean erabiltzen dituzte erreferentzia teoriko eta metodologiko batzuek, eta, betiere, berauen gizartelangintza komunitarioko eskuartzea bideratu eta orientatzen dute indibiduo eta komunitateen sistema natural eta komunitarioetara, eta horien arteko bat da sare sozialetako eskuartzea. Artikulu honetan aztertzen dira oinarri etiko eta teknikoak, dimentsio teoriko eta metodologikoak, eta baita ere sare sozialetako eskuartzeetan gizarte-langileek izaten dituzten gaitasun zientifiko eta teknikoak. Horretarako oinarri bilakatzen dira Madrilgo Erkidegoko (Espainia) ehun mila biztanle baino gehiago dituzten udaletako gizarte-langileekin buruturiko erdi-egituratutako elkarrizketak. Bertan buruturiko analisiaren ondoriotzat uler daiteke tokiko gizarte-langintza komunitarioak beharrezko dituela alderdi indartsuetan oinarritzen diren ikuspegiak, eta ikuspegi horiek nabarmendu behar dutela sare sozialak sortu eta indartzearen garrantzia, bide horretatik baliabideak bideratu ahal izateko aldaketa egoki baten norantzan.

\section{GAKO-HITZAK:}

Sare sozialak, oinarriak, dimentsioak, gaitasunak, gizarte-langintza komunitarioa.
Los trabajadores sociales utilizan, en sus prácticas profesionales, diferentes referentes teóricos y metodológicos, basados siempre de los sistemas naturales y comunitarios de los individuos y comunidades hacia los que dirigen y orientan su intervención en trabajo social comunitario, siendo uno de ellos la intervención en redes sociales. Este artículo analiza los fundamentos éticos y técnicos, las dimensiones teóricas y metodológicas, y las competencias científicas y técnicas del trabajador social en la intervención en redes sociales, a partir de los resultados de entrevistas semiestructuradas realizadas a trabajadores sociales de los ayuntamientos de la Comunidad de Madrid (España) con más de cien mil habitantes. Del análisis, cabe inferir que el trabajo social comunitario en el ámbito local necesita enfoques basados en las fortalezas para trabajar con las comunidades, enfoques que enfaticen la construcción y el fortalecimiento de las redes sociales, para que se movilicen sus recursos hacia un cambio positivo.

\section{Palabras Clave:}

Redes sociales, fundamentos, dimensiones, competencias, trabajo social comunitario. 


\section{Introducción}

En los últimos años, se han producido cambios importantes en la organización social que obligan al trabajo social a transformar su práctica, tales como el incremento de las relaciones efímeras, que ha provocado un debilitamiento de los vínculos de inserción comunitaria (Bonet, 2006; Pérez Díaz, 2008) y de los procesos de individualización (Bauman, 2011), además de un agravamiento/ aumento de las situaciones de pobreza y exclusión social (De Robertis, 2000) y de los problemas relativos a las condiciones de vida, es decir, ligados a las desigualdades de género, a la inmigración y al envejecimiento (Giddens, 2007) Se trata de cambios cuyas consecuencias suponen nuevos desafíos para el trabajo social comunitario, por tratarse de problemas que reclaman acciones comunes desde un abordaje colectivo, cuyo método debe contribuir a la generación del capital social de las personas. Un concepto inherente a la estructura de las relaciones entre dos o más personas es el de capital social (Coleman, 1988; Putnam, 2003), y la generación del capital social relacional es decisiva para el trabajo social, porque supone un compromiso con la mejora de las capacidades de las personas que le permitan restablecer los vínculos sociales (el vínculo de uno/a mismo/a con la sociedad y el vínculo del nivel comunitario con el nivel social), creando así las condiciones necesarias para activar, sustituir y complementar las redes sociales en las que las personas se encuentran inmersas (Bonet, 2006; De Robertis y Pascal, 2007). Es importante subrayar, como afirma Gilchrist, que "los vínculos entre las personas y las organizaciones son una parte fundamental de la capacidad de una comunidad para actuar colectivamente y participar en la toma de decisiones en los asuntos públicos" (2009: 123).

Por otra parte, la finalidad del trabajo social es la lucha contra la exclusión social y gran parte de las exclusiones actuales están vinculadas a la vulnerabilidad de las relaciones en las redes sociales personales, los sistemas de apoyo y la escasez de vínculos de inserción comunitaria, lo que requiere de los trabajadores sociales la competencia necesaria para comprender una realidad compleja, con una pluralidad de interacciones, y también conocimientos sobre los distintos problemas sociales que les ayuden en el conocimiento de las fortalezas, las dificultades en las que intervienen y en la utilización de los diferentes recursos, que, según señala Del Pino (2000), pueden ser personales (la capacidad de reaccionar y de afrontar los problemas), ambientales (la capacidad de entrar en contacto y utilizar los sistemas de ayuda natural, las redes de apoyo social) y sociales (los recursos institucionales organizados en servicios, programas y prestaciones). No podemos dejar de señalar, igualmente, que la intervención en el ámbito local parte de un contexto de proximidad que facilita un conocimiento muy importante de la realidad social, que convierte al territorio en un eje fundamental de la intervención social centrada en la inclusión social, desde donde se debe impulsar el trabajo en red, y que ofrece grandes oportunidades si va unida a dos factores que se consideran, a menudo, muy importantes en el abordaje de los problemas sociales:

el contexto de proximidad en que se desenvuelve la acción, desde una perspectiva de conocimiento más profundo de la realidad social y la posibilidad de establecer relaciones con los afectados por los problemas, así como con las entidades de red social local, desde una óptica de intervención que puede darles respuesta (Cardoso, 2012: 183).

Para responder a la realidad descrita, los trabajadores sociales necesitan teorías que les faciliten categorías de pensamiento y elaborar modelos de intervención que les permitan abordar el análisis de la acción, en nuestro caso, desde el enfoque de redes sociales, porque no cuentan con un referente teórico y metodológico que, partiendo de sus prácticas profesionales concretas, integre la teoría y los conceptos de dicho enfoque, instrumento que consideramos puede servir de ayuda para analizar eficazmente las relaciones sociales de las personas y los diferentes recursos de sus redes sociales. En el presente trabajo, queremos avanzar en la comprensión de las intervenciones en redes sociales que realizan los profesionales del trabajo social, mediante el análisis de:

- Los fundamentos éticos y técnicos del trabajo social comunitario en la intervención en redes sociales.

- Las dimensiones teóricas y metodológicas del trabajo social comunitario en redes sociales.

- Las competencias científicas y técnicas del trabajador social en la intervención en redes sociales.

\section{Fundamentos éticos y técnicos del trabajo social comunitario en la intervención en redes sociales}

El trabajo social contempla, en su génesis, un conjunto de principios y fundamentos de los derechos humanos y una ética del compromiso con la ciudadanía, que promueve el bienestar social y la protección social, con el objetivo de mejorar la calidad de vida de los ciudadanos. En este sentido, "los trabajadores sociales deben continuar con la búsqueda de nuevas maneras de ayudar, situando siempre sus esfuerzos a partir de la consideración de los valores y la ética de la profesión" (Reamer, 2013: 171). En relación con los fundamentos éticos del trabajo social comunitario, la dimensión de los valores se considera central en trabajo social, porque querer ayudar a las personas es un elemento fundamental en su práctica, pero se necesita igualmente el compromiso con la justicia social, la integridad y la competencia profesional, siendo "la combinación entre valores y ética lo que hace único al trabajo social entre las diferentes profesiones de 
ayuda" (Segal, Gerdes y Steiner, 2013: 18). Se trata de una dimensión tanto más presente (Aballéa, Ridder y Gadéa, 2003) cuanto más al límite se sitúan las situaciones de intervención, en términos de injusticia o sufrimiento del otro, de cuestionar su existencia como sujeto, o aspectos existenciales que muestran la cara más vulnerable de la condición humana. Por otra parte, los valores que dan sentido a los principios éticos del trabajo social son valores democráticos, presentes en todas las Constituciones de los países considerados avanzados, lo cual no obsta para que sea necesario promocionarlos en el ámbito de la intervención social, pues ahí es donde comúnmente se manifiestan las mayores trasgresiones de estos principios éticos, valores supremos que crecen en defensa de la dignidad humana y que precisan de una estrategia para su inserción en la profesión de trabajador social. Los trabajadores sociales tienen como fundamento de su intervención los valores profesionales y los principios éticos, pero también otros aspectos decisivos, como la experiencia profesional adquirida, la bibliografía, la supervisión, la información sobre legislación, las tecnologías de la información y la comunicación, el trabajo en coordinación con otros profesionales, y los conocimientos teóricos y metodológicos.

Con respecto a los fundamentos técnicos, es necesario hacer efectivo el empowerment, que implica un trabajo de capitalización de los sujetos en sus trayectorias individuales y colectivas en que se encuentran debilitados, sin olvidar tampoco que "lo que vertebra el trabajo comunitario es, sin lugar a dudas, el elemento de la participación" (Marchioni, 2001: 10). El empowerment, según diferentes autores (Lee, 2001; Adams, 2008), es un abordaje integrador, holístico y adecuado a las necesidades de la población; un marco conceptual utilizado para guiar la práctica de trabajo social, al que Song (2011) considera un objetivo intermedio en las diferentes áreas de intervención del trabajo social, que persigue fortalecer a los usuarios para que puedan romper la situación de desventaja social en la que se encuentran y, a su vez, mejorar su calidad de vida, aumentando su participación social y la utilización de los recursos existentes. Para Folgheraiter (2012), es una noción controvertida, pero fundamental para entender hasta el fondo el trabajo social de red. Según dicho autor, empowerment es un proceso que, desde el punto de vista de quien lo experimenta, significa 'sentir que tengo poder', o 'sentir estar en condiciones de poderlo tener', y desde el punto de vista de quien lo hace posible, significa "una actitud técnica capaz de acrecentar la probabilidad de que las personas se sientan en grado de hacer" (Folgheraiter, 2012: 405). Frans (1993) considera el empowerment un concepto unitario que describe el proceso y la meta hacia la que orientar los esfuerzos de corrección de la falta de poder. Desde nuestro punto de vista, dicho concepto implica diferentes dimensiones (Rodríguez, 2014b)

- Ayuda a entender el trabajo en red, porque promueve la participación de la ciudadanía y la construcción de la sociedad civil.
- Contribuye a generar respuestas creativas a los problemas por parte de los diferentes recursos existentes.

- Impulsa procesos de desarrollo y crecimiento de las personas.

- Produce nuevos conocimientos y nuevas competencias, de forma que cuantos más y más fuertes vínculos tiene una persona, mayor acceso tendrá a los recursos.

\section{Dimensiones teóricas y metodológicas del trabajo social comunitario en la intervención en redes sociales}

Los profesionales del trabajo social deben contar con distintas referencias teóricas y metodológicas, porque la mayoría de los problemas que tienen que abordar no nacen de un déficit humano o social en sí mismos, sino que son resultado de la interacción entre lo humano y lo social, dado que "en muchas situaciones concretas de la vida, un déficit humano no produce necesariamente un problema objetivamente relevante, salvo que se den al mismo tiempo otras realidades que vienen del medio social" (Folgheraiter, 2012: 61). Por tanto, podemos considerar que los trabajadores sociales intervienen de forma prioritaria en el ámbito local con individuos, familias y colectividades con problemas derivados de encontrarse en una situación de dificultad social sin red de apoyo o red muy escasa; con personas en situación de aislamiento físico y social (mayores solos, inmigrantes sin red, retornados); con personas que viven una situación de sobrecarga de algún miembro en la función doméstica, sin red que les apoye; o bien con personas en proceso de rehabilitación con poco apoyo social.

No podemos dejar de señalar que los riesgos a los que se enfrentarán los ciudadanos del siglo XXI no son tan sólo los 'antiguos riesgos' frente a los que solía protegernos el Estado del bienestar tradicional (como el desempleo o la vejez), sino también 'riesgos nuevos', porque los numerosos cambios producidos en la organización social, en la constitución de la familia y en los procesos de socialización, entre otros muchos, han producido a lo largo de los años una sociedad marcadamente individualista y vincularse se ha convertido en un riesgo para las personas, pues la inseguridad y el riesgo no crean vínculos sólidos de comunidad. En la actualidad, la pobreza y la exclusión social constituyen problemas sociales que únicamente pueden ser comprendidos desde el análisis de las dinámicas históricas de las sociedades que los producen, y el término exclusión social hace referencia a "un proceso de pérdida de integración o participación del individuo en la sociedad en uno o varios ámbitos (económico, político, social-relacional), siempre en términos relativos a su situación con respecto al conjunto de la población” (Arnaut-Bravo, 2010: 37). Ahora bien, mediante el trabajo social comunitario, que capacita a las personas, se pueden afrontar las características 
estructurales de nuestras sociedades para orientar los procesos de cambio social en una dirección determinada, y los trabajadores sociales (Dominelli, 2009) deben facilitar procesos de cambio que vayan en la dirección de lograr una mayor equidad social. Marshall considera que son tres "los elementos significativos de la tradición profesional que hay que rescatar: singularidad y atención personalizada, dimensión social de la persona y análisis de la situación para orientar la intervención" (2006: 181). Para Villalba (1995), el trabajo con redes con personas individuales y con familias requiere la distinción de tres fases: identificar la red, analizar la red e intervenir en red. Cuando hablamos de identificar la red social (Rodríguez, 2014: 303), nos referimos a conocer la existencia de personas de la comunidad que las ayudan; a la capacidad de autocuidado de la persona o familia de referencia y de los miembros de la red más relevantes; a los profesionales que las están ayudando desde el sistema formal y con quienes han establecido una relación de ayuda y confianza; y a las posibilidades de las personas para cuidar, mantener, aumentar o modificar su propia red.

\section{Competencias científico-técnicas de la intervención del trabajo social comunitario en la intervención en redes sociales}

Un reto importante del trabajo social es aumentar su comprensión de las fortalezas y los problemas de las comunidades en las que interviene, siendo la capacidad de una profesión de definir su objeto de intervención una condición esencial para su reconocimiento científico, al igual que el conocimiento de teorías, métodos y modelos y "su utilización científica y continuada, que contribuirá de forma decisiva a un proceso de redefinición, puntualización, reflexión y crecimiento del saber del trabajo social" (Viscarret, 2007: 78). Los trabajadores sociales utilizan diferentes modelos y teorías en su intervención profesional. Se trata de modelos compatibles entre sí, que pueden utilizarse de manera complementaria, tales como el modelo ecológico y de sistemas, la teoría de la comunicación humana y el enfoque de redes sociales, que permite crear las condiciones para potenciar, sustituir 0 complementar los sistemas de apoyo comunitario. Haciendo referencia a la teoría de redes, Folgheraiter (2012: 29) destaca la importancia de "poder contar con un paradigma alternativo capaz, por fin, de 'narrar' e interpretar nuestros códigos desde la (relativa) autonomía de los diferentes agentes"; y para Villalba (2009), el trabajo de y en redes se considera hoy un paradigma global desde la perspectiva ecológica, que interconecta los distintos niveles de intervención (individual-familiar, grupal y comunitario), teniendo en cuenta la participación de los usuarios en sus propios procesos de ayuda y también las dinámicas del sistema formal (instituciones, equipos) y de los sistemas informales de ayuda, que provienen de los propios contextos relacionales de los usuarios.
No podemos hablar de competencias científicotécnicas del trabajador social sin referirnos, de manera obligada, a los diferentes roles que puede asumir y las diferentes funciones que realiza en su intervención en redes sociales. Las capacidades y habilidades profesionales que se requieren en la intervención en redes se refieren a la articulación y el fortalecimiento de los diferentes tipos de relaciones, ya sean personales, sociales, intergrupales o interinstitucionales, entre otras. Siguiendo a Ferrario (2009), en las intervenciones en redes el trabajador social puede asumir los roles de experto-consultor, orientador-formador, referente organizativo y de recursos, facilitador de procesos individuales y colectivos, o coordinador de ayudas externas (Rodríguez, 2014b: 309) y, según refiere Chopart (2003), las funciones del trabajo social (principalmente la acogida, la orientación y el acompañamiento) continúan siendo la mejor armadura para la intervención social. Con respecto a la intervención en redes sociales, el trabajador social tiene, entre sus funciones, (Rodríguez, 2014b: 311) identificar el tejido relacional y conocer los recursos existentes en la comunidad; examinar, junto con la persona/familia, las redes de apoyo a las que puede acceder; contribuir al desarrollo y evaluación de las redes de apoyo; complementar los recursos de apoyo existentes en las redes de las familias; mediar en las interacciones conflictivas de las redes; y mediar en la relación entre las personas y sus sistemas de apoyo.

En este punto de nuestra investigación, es necesario subrayar que la intervención en redes sociales necesita nuevas respuestas instrumentales, porque los trabajadores sociales utilizan distintas técnicas, en consonancia con el enfoque holístico de intervención con las personas y la sociedad, cuya aplicación requiere siempre la competencia en su creación, selección y práctica posterior. Los trabajadores sociales pueden utilizar diferentes técnicas en su intervención en redes sociales (Rodríguez, 2014b: 346): en relación con el estudio y el diagnóstico social (mapa de red social, atención personal, contacto telefónico, visita domiciliaria, entrevista, observación, técnicas documentales, reuniones); en relación con la intervención social de manera específica (informe social, reuniones, mediación individuo/ familia y comunidad, programa de inserción, diseño de la propia red profesional, intervención en red comunitaria, asamblea); y en relación con la evaluación de los objetivos señalados (entrevista, mapa de red anterior a la intervención y mapa de red actual), que nos permitan verificar los cambios producidos, y a veces, modificar los propios objetivos.

\section{Metodología}

En el presente artículo, se presentan las respuestas parciales de la investigación empírica realizada en 2013 y que forma parte de una tesis doctoral (Rodríguez, 2014b) en relación con los fundamentos éticos y técnicos, las dimensiones teóricas y metodológicas, y las competencias científicas y 
técnicas del trabajador social en la intervención en redes sociales.

El uso de diferentes técnicas de recogida de información para contrastar los datos obtenidos es una estrategia clave para aumentar la capacidad analítica y la validez de la investigación. Así, hemos utilizado como procedimientos metodológicos el análisis documental, las entrevistas semiestructuradas realizadas a trabajadores sociales y el análisis de su contenido, organizado en categorías basadas en la articulación de los elementos teóricos con elementos empíricos.

Con respecto a la elección de la entrevista semiestructurada, podemos considerar que "la entrevista en profundidad focalizada o semiestructurada es una técnica de investigación sociológica, ampliamente utilizada, que permite realizar un análisis de carácter cualitativo estructural, dirigida a obtener información sobre un asunto concreto y, por ello, va dirigida a un individuo concreto, caracterizado y señalado previamente por haber tomado parte en esa situación o haber vivido esa experiencia” (Ruiz e Ispizua, 1989: 153-154). Ruiz e Ispizua (íbid.), retomando los planteamientos de Merton, destacan como características de la entrevista focalizada que la persona entrevistada es un sujeto que ha intervenido en una situación particular; que el entrevistador conoce de antemano, directa o indirectamente, esta situación; que la entrevista se concentra en la experiencia subjetiva de los sujetos seleccionados, con el objeto de conseguir de ellos su definición de la situación; y que está siempre abierta a que respuestas o relatos imprevistos den pie a nuevas hipótesis o interpretaciones de la experiencia. Tal como señalan Báez y Pérez de Tudela (2007: 102), "en la entrevista semiestructurada se trabaja en unos contenidos y un orden preestablecido, dejando abierta la gama de posiciones que desee manifestar el entrevistado". La elección de dicho método de recogida de datos nos va a permitir verificar en el campo empírico la información recogida en el marco teórico y, al mismo tiempo, explorar el discurso de los entrevistados, buscando nueva información de otros aspectos que pueden no haber sido contemplados en el análisis documental y que viven los profesionales en su práctica profesional.

Las características de la metodología cualitativa (Cea, 1996) se pueden resumir en que trabaja con grupos o muestras estructurales, no con muestras estadísticamente representativas. En el presente estudio, la selección de la muestra de los trabajadores sociales se realizó teniendo en cuenta los siguientes criterios: que prestaran sus servicios en centros de servicios sociales de ayuntamientos de la Comunidad de Madrid cuya población superara los cien mil habitantes (Alcalá de Henares, Alcobendas, Alcorcón, Fuenlabrada, Getafe, Leganés, Móstoles, Parla y Torrejón de Ardoz), y que contaran con al menos diez años de experiencia como trabajadores sociales en la administración local. Buscábamos una muestra cuya información fuese extrapolable a la totalidad de los trabajadores sociales que prestan sus servicios en la administración local, y consideramos relevante la experiencia laboral de los profesionales en el ámbito de la atención primaria, puesto que, "hace que las opiniones recibidas se sustenten en un amplio conocimiento por parte de los profesionales, así como en su capacidad para detectar debilidades, amenazas, fortalezas y oportunidades del sistema" (Subirats, 2007: 60); e igualmente recoger la especificidad de municipios diferentes, porque los servicios desarrollados con un enfoque comunitario permiten reconocer la diversidad de las personas y del territorio en el que se producen las desigualdades y emergen nuevos riesgos de vulnerabilidad. La especificidad de cada espacio social y territorial es un factor determinante para el desarrollo de intervenciones sociales y objeto de interés en nuestra investigación.

Diversos autores (Guba y Lincoln, 1982; Goetz y Lecompte, 1988) han manifestado reiteradamente en sus escritos que entre veinticinco y treinta entrevistas sería el ideal para una investigación de este corte, ya que a partir de treinta, se saturan los datos. Por lo tanto, consideramos que un número similar sería significativo para lo que se consideran los cánones científicos de nuestra investigación. El muestreo finaliza cuando dejan de surgir elementos o incidentes nuevos con significación propia; por ello, el concepto clave es el de saturación del discurso. En nuestro caso, la muestra estaba constituida por treinta trabajadores sociales, y a pesar de que se recibieron más cuestionarios, no se incorporaron al estudio, porque se había alcanzado ya la saturación teórica, al no añadir el resto de cuestionarios información relevante sobre el objeto de estudio, ni realizar nuevos planteamientos.

El cuestionario constaba de distintas preguntas. La mayoría pedía responderlas mediante un baremo de importancia (1, 'nada importante'; 2, 'poco importante'; 3, 'bastante importante'; y 4, 'muy importante'); también se daba la posibilidad de no elegir ninguna de las opciones señaladas, y todas las preguntas incorporaban un apartado titulado 'otros' con el objeto de ofrecer al entrevistado la oportunidad de matizar su respuesta o dar una respuesta alternativa que considerase igualmente importante. Una pregunta sobre la intervención profesional admitía respuesta múltiple (con cuatro opciones) e invitaba a añadir otras respuestas diferentes. También había una pregunta abierta que indagaba sobre los problemas sociales comunitarios existentes en el municipio donde realizaban su actividad profesional y que necesitarían ser abordados mediante la intervención en redes sociales.

Para el análisis cuantitativo estadístico-descriptivo de las respuestas, se utilizó una metodología cuantitativa, a través del software Statistical Package for Social Sciences (SPSS 20), que nos sirvió de soporte para la codificación y análisis de resultados. 


\section{Resultados}

En relación a los fundamentos éticos y técnicos del trabajo social comunitario en la intervención en redes sociales, hemos preguntado a los trabajadores sociales cuáles son los fundamentos de su intervención en redes sociales (Gráfico 1), los aspectos prioritarios en su intervención en redes sociales (Gráfico 2) y el concepto de empowerment (Gráfico 3). Los resultados obtenidos se exponen a continuación.

Los trabajadores sociales han señalado como fundamento de su intervención en redes sociales en el ámbito local, en primer lugar, los valores y los principios éticos, y en segundo, la experiencia profesional; en cambio, en sus respuestas han aparecido raramente como tales la bibliografía y la supervisión. Sin embargo, consideramos que la supervisión y los programas de desarrollo de personal realizados de manera sistemática podrían cultivar un ambiente de aprendizaje eficaz que ayudara a los profesionales en los procesos de toma de decisiones éticas y que incrementara su competencia profesional, porque la experiencia práctica que ignora la teoría y la evidencia sistemática puede incluso caer en el prejuicio (Unwin y Hogg, 2012) y, ciertamente, disminuye la potencial capacidad de los trabajadores sociales para ser agentes efectivos del cambio.

Los trabajadores sociales orientan su intervención profesional con individuos/familias en el ámbito de las redes sociales hacia 'el trabajo con las capacidades y competencias de los individuos/ familias' (98\%), lo cual compartimos, porque consideramos que la relación de ayuda debe partir de la valoración de las competencias personales, para ganar autonomía y ciudadanía plena. Sin

Gráfico 1. Fundamentos de la intervención del trabajador social en redes sociales (\%)

Bibliografía
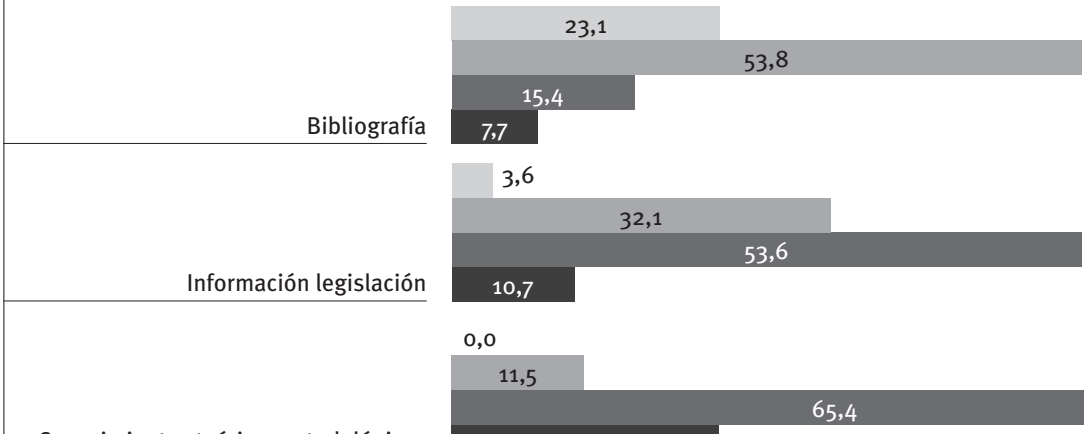

Conocimientos teórico-metodológicos
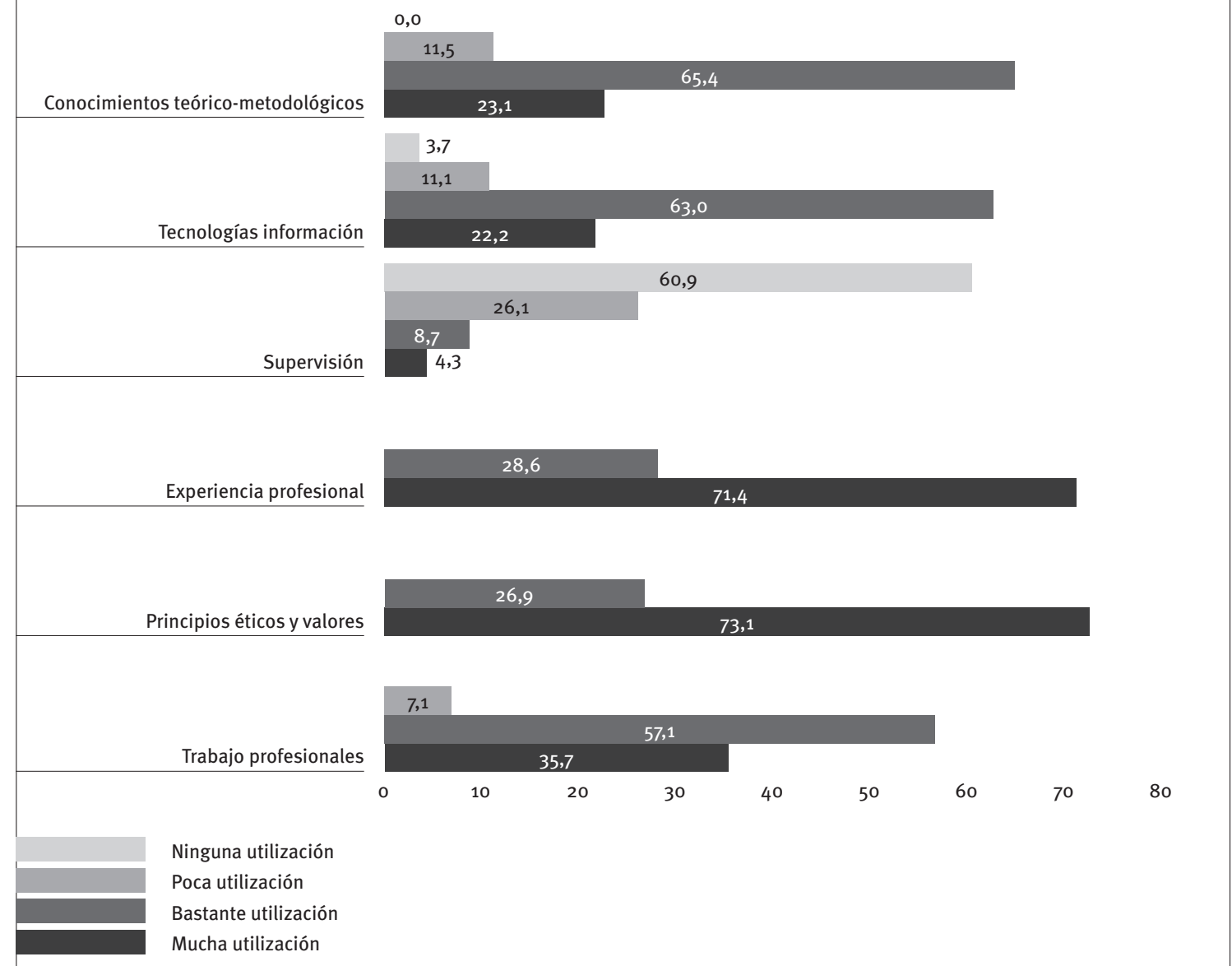

Fuente: Rodríguez (2014b). 
Procedimientos teóricos y tecnico operativos

66,6

Trabajo con capacidades/competencias individuos

Utilización respuestas redes sociales

Obtención recursos/medios disponibles comunidad

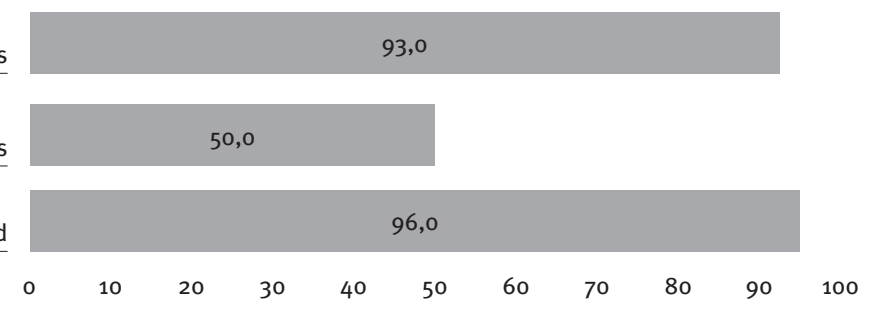

Fuente: Rodríguez (2014b).

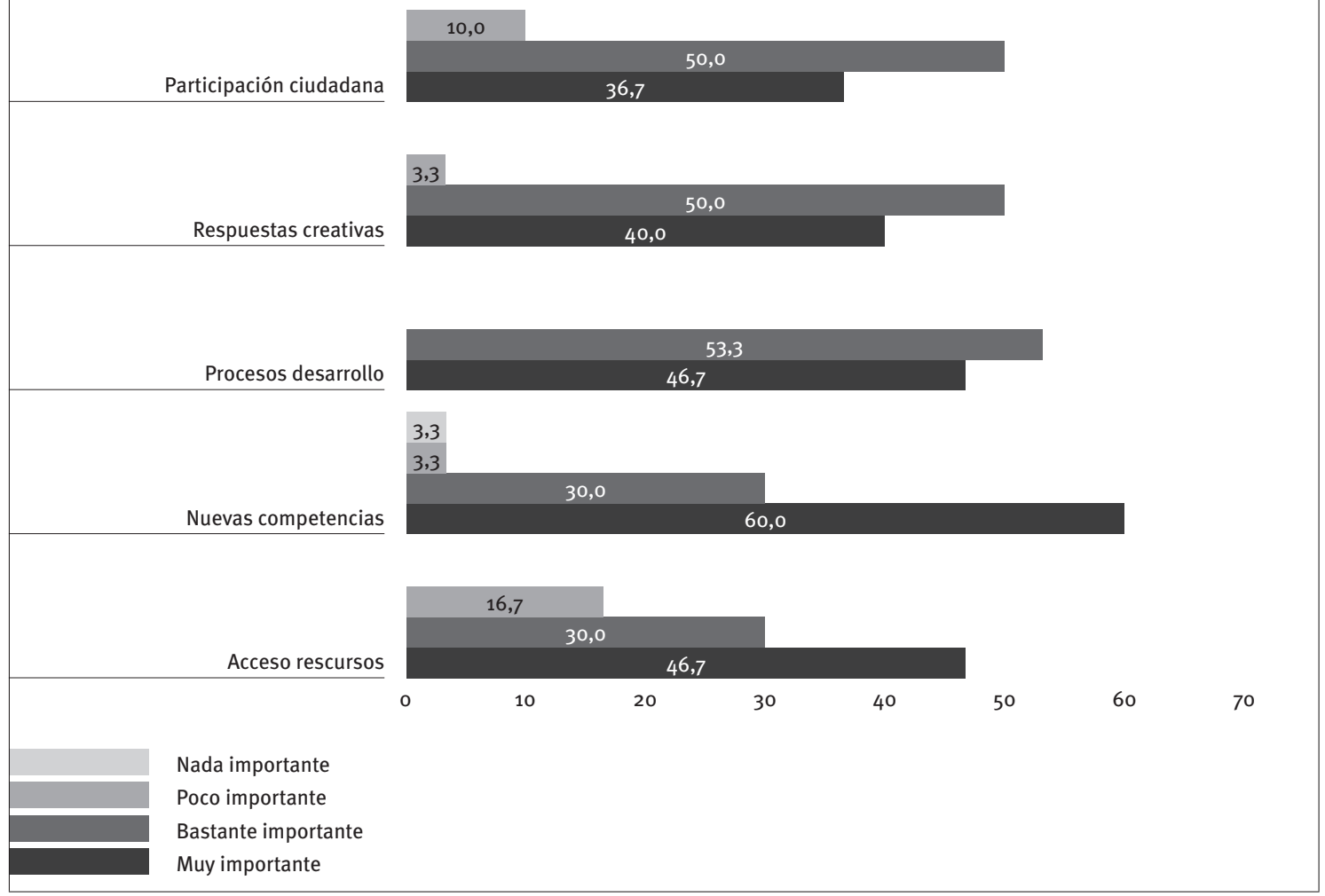

Fuente: Rodríguez (2014b).

duda, defendemos la intervención que tiene como objetivo modificar el ambiente de tal forma que se den las condiciones humanas, sociales y físicas que respondan a las necesidades de las personas. En este sentido, la referencia al 'fortalecimiento' (empowerment) invita a un modo de trabajar que implica a las personas, y promueve la posibilidad de influir en el ambiente, constituye, por tanto, el instrumento fundamental para intervenir en redes. Destacamos igualmente que un $86 \%$ de los trabajadores sociales han señalado como un aspecto prioritario de la intervención con redes 'la obtención de recursos o medios disponibles en la comunidad para la intervención concreta que realiza', y que únicamente un $50 \%$ utiliza 'las respuestas que ofrecen las redes sociales en general'.

Los trabajadores sociales han considerado muy relevantes (Gráfico 3 ) todas las afirmaciones relativas al concepto de empowerment de los individuos/ familias/colectividades con los que trabajan, especialmente: 'se obtienen nuevos conocimientos y se desarrollan nuevas competencias' e 'impulsa procesos de desarrollo y crecimiento de las personas'. Con la conciencia de estar en red, las personas llegan a sentir que pueden decidir en relación con las respuestas, o crearlas desde una reflexión común, y “así nace, o se refuerza más 
aún, el empowerment entendido como el sentido de poder hacer, de tener la posibilidad de actuar con la 'potencia' necesaria en la solución al problema” (Folgheraiter, 2011: 579).

Con respecto a las dimensiones teóricas y metodológicas del trabajo social comunitario en la intervención en redes sociales, hemos preguntado a los trabajadores sociales sobre el tipo de problemática que presentan los individuos/familias/colectividades considerados de intervención prioritaria en redes sociales por los trabajadores sociales, para así conocer mejor a quién se dirige su intervención (Gráfico 4), los problemas sociales comunitarios más importantes existentes en el municipio donde realizan su actividad profesional (Cuadro 1) y la fase de identificación de la red social (Gráfico 5).

Los $46,7 \%$ trabajadores sociales han considerado muy importante la 'dificultad social sin red de apoyo o muy escasa' (46,7\%, muy importante; $23,3 \%$, bastante importante) y el 'aislamiento físico y social (mayores solos, inmigrantes sin red, retornados)'. Un trabajador social considera de intervención prioritaria las "familias de todo tipo sin recursos, con compromiso de pago y con la red de apoyo agotada por lo prolongado de su situación" y otro ha señalado "las cuestiones de género y los medios de comunicación”.

Los programas de intervención comunitaria se elaboran a partir del diagnóstico social de una comunidad, diagnóstico en el que están presentes, según afirma Ferreira (2011), algunos elementos de orden personal y familiar, tales como incapacidades y competencias insuficientes, y otros elementos de orden social y económico, como la precariedad económica o el desempleo.

Con respecto a las relaciones efímeras (debilidad de los vínculos de inserción comunitaria) y los procesos

Gráfico 4.Tipo de problemática que presentan los individuos/familias/colectividades considerados de intervención prioritaria en redes sociales por los trabajadores sociales (\%)

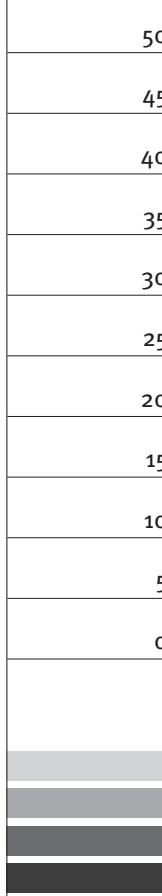

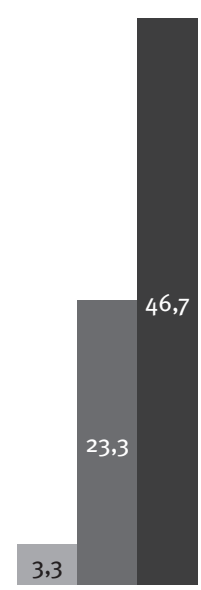

Dificultad social

Nada importante

Poco importante

Bastante importante

Muy importante

Fuente: Rodríguez (2014b).

\begin{tabular}{|l|l|}
\hline \multicolumn{2}{|l|}{ Cuadro 1. Problemas sociales comunitarios que necesitarían ser abordados desde la intervención en redes sociales } \\
\hline Problemas sociales comunitario & Trabajadores sociales \\
\hline $\begin{array}{l}\text { Relaciones efímeras (debilidad de los } \\
\text { vínculos inserción comunitaria) }\end{array}$ & $\begin{array}{l}\text { Soledad, falta de apoyo mutuo, falta de concienciación de la realidad social, falta/escasa } \\
\text { relación vecinal y ruptura/ausencia de red. }\end{array}$ \\
\hline Procesos de individualización & Aislamiento social, incapacidad de pedir ayuda. \\
\hline Exclusión social & Ausencia de garantía de los ingresos mínimos, nuevos grupos en riesgo de exclusión. \\
\hline $\begin{array}{l}\text { Condiciones de vida (género, inmigración, } \\
\text { envejecimiento y modelos de vida } \\
\text { ecológicos) }\end{array}$ & $\begin{array}{l}\text { Aislamiento social de los mayores, conciliación de la vida laboral y familiar, familias } \\
\text { con dependientes a cargo y sobrecarga física y emocional, madres jóvenes, familias } \\
\text { monoparentales, falta de apoyo a madres solas, dificultades de integración de la población } \\
\text { inmigrante. }\end{array}$ \\
\hline
\end{tabular}

Fuente: Rodríguez (2014b). 
de individualización, los trabajadores sociales han destacado especialmente las situaciones de soledad, los problemas de ruptura/ausencia de red y el aislamiento social. En lo relativo a la pobreza y la exclusión social, han destacado la ausencia de garantía de ingresos mínimos y la existencia de nuevos grupos en riesgo de exclusión social.

En la fase de identificación de la red social, los trabajadores sociales han considerado necesario conocer todos los aspectos señalados, y en particular, 'la existencia de personas de la comunidad que les ayudan' (muy importante para el 69,2 \% y bastante importante para el $26,9 \%$ ) y 'la capacidad de autocuidado de la persona o familia de referencia y de los miembros de la red más relevantes'. Las redes sociales contribuyen a ampliar los límites de la acción social, y en el trabajo social comunitario, se requiere la detección, la identificación y el estudio de una variedad muy amplia de factores influyentes en una situación determinada.

Por último, presentamos los resultados en relación con las competencias científicas y técnicas de la intervención del trabajo social comunitario en la intervención en redes sociales; en concreto, hemos preguntado a los profesionales sobre los modelos que utilizan en su intervención profesional (Gráfico 6), los roles que desempeñan (Gráfico 7), las funciones que realizan en su intervención en redes sociales (Gráfico 8) y las técnicas específicas que utilizan (Gráfico 9).

\section{Gráfico 5. Identificación de la red social (\%)}

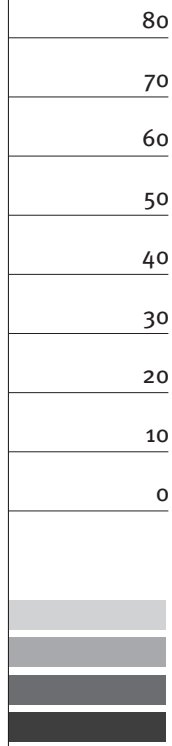

60

50

40

30

Capacidad de autocuidado persona

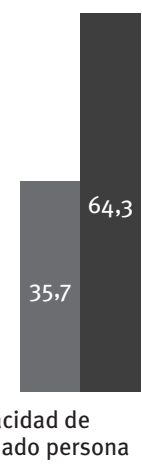

Nada importante

Poco importante

Bastante importante

Muy importante

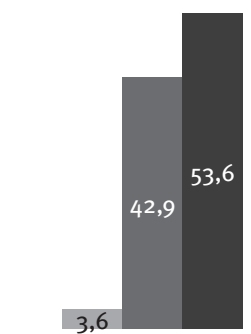

Posibilidades cuidar, aumentar o modificar red

Fuente: Rodríguez (2014b).

\section{Gráfico 6. Valoración de la utilidad de los modelos por parte de los trabajadores sociales (\%)}

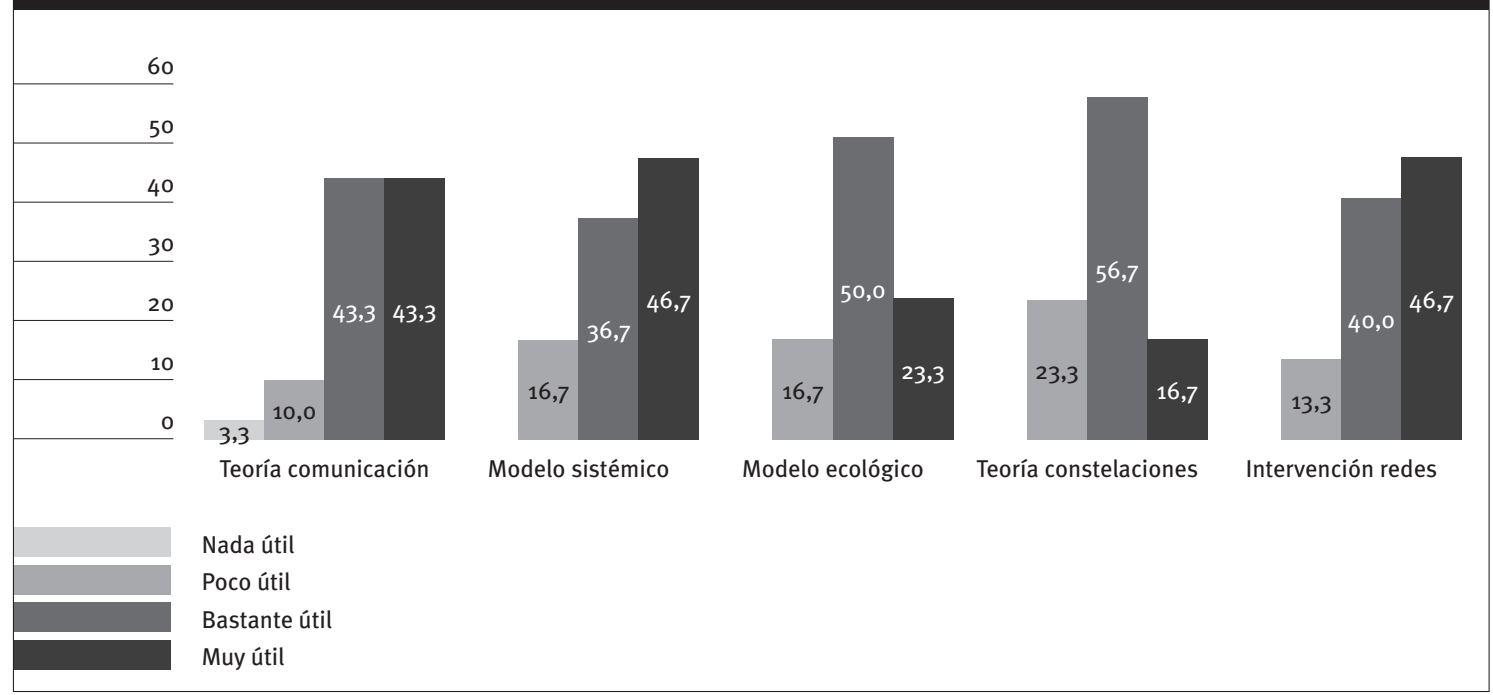

Fuente: Rodríguez (2014b). 


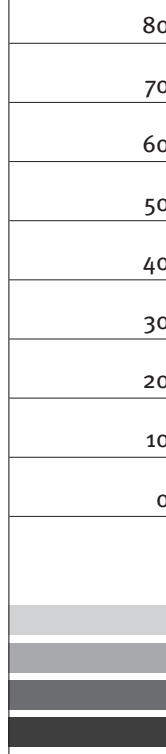

80

70

60

50

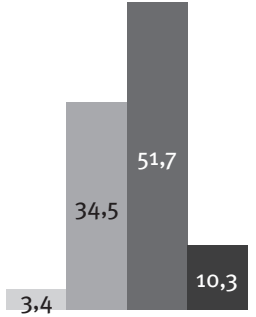

Experto consultor

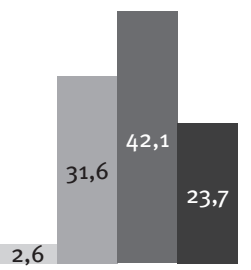

Referente organizativo

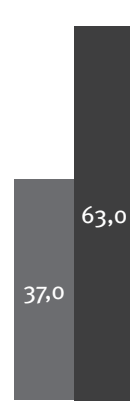

Facilitador procesos

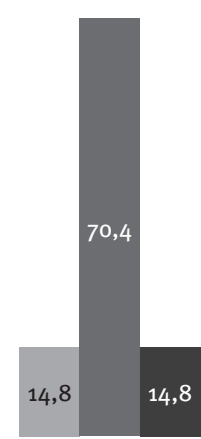

Orientador-formador

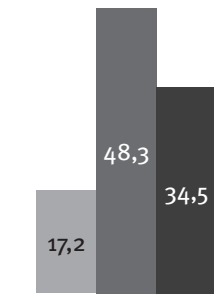

Coordinador ayudas

Nada importante

Poco importante

Bastante importante

Muy importante

Fuente: Rodríguez (2014b).

La mayoría de los trabajadores sociales participantes en la investigación han identificado los referentes teórico-metodológicos que coinciden con el modelo sistémico y la intervención en redes sociales como soportes teóricos principales de su intervención. La aplicación de uno u otro modelo depende de lo que requiera cada situación y el contexto en el que se sitúe.

Los trabajadores sociales han valorado en un $63 \%$ como muy importante y un $37 \%$ como bastante importante el rol de 'facilitador de procesos individuales y colectivos'; sólo un 10,3\% ha considerado muy importante el de 'experto consultor' y un $34,5 \%$ lo ha considerado poco importante; por su parte, el 31,6\% ha considerado poco importante ser 'un referente organizativo y de recursos'.

El Gráfico 9 nos muestra que los trabajadores sociales han considerado la importancia de todas las funciones señaladas, pero han destacado como muy importantes las de 'identificar el tejido relacional y conocer los recursos existentes en la comunidad' $(72,4 \%)$, 'examinar con la persona/familia las redes de apoyo a las que pueden acceder' $(71,4 \%)$ y 'contribuir al desarrollo y evaluación de las redes de apoyo’ (69\%). Del análisis del Gráfico, se desprende que las funciones del trabajador social en el ámbito de las redes sociales están relacionadas con las redes, pero no tanto con las técnicas específicas de intervención.

Las técnicas más utilizadas en la intervención en la red social desde el nivel individual/familiar son 'el informe social', 'la atención personal', 'las reuniones’ y ‘la mediación individuo/familia y comunidad'. A este respecto, constatamos la enorme diferencia que existe con 'la intervención en red comunitaria', utilizada únicamente por el 36,7\% de los profesionales, y 'las asambleas', empleadas sólo por el 3,3\%, lo cual refleja que la intervención que se realiza es de tipo individual frente a otra de carácter más global. El Gráfico 10 nos muestra que los trabajadores sociales utilizan técnicas específicas de intervención en red social, como 'el mapa de red', pero si comparamos estos resultados con los del Gráfico 8, vemos que entienden el trabajo en red y lo realizan desde una perspectiva técnica, pero sin embargo, en las funciones se sitúan, en parte, desde un punto de vista de gestión, es decir, desde la dependencia institucional, por lo que desarrollan roles, como el de 'coordinador de ayudas externas', que se dirigen más hacia la parte burocrática derivada de su rol institucional, porque, en ocasiones, "el trabajador hace un diagnóstico desde los servicios que dispone y no desde el origen de la necesidad, es decir, desde lo que pide la propia realidad" (Folgheraiter, 2012: 401). En este mismo sentido, cuando nos referimos a organismos públicos, "la flexibilidad es más difícil de programar y será necesario una sistematización diferente de la autonomía profesional a nivel interno, así como una valoración más decidida" (ibíd.: 403).

\section{Conclusiones}

A lo largo del presente trabajo, hemos sistematizado la información que nos permitiese avalar las condiciones en que se desarrolla la intervención en redes sociales en el ámbito local, realizando una serie de avances teóricos y metodológicos relativos a la integración práctica de los conceptos y la teoría del enfoque de la intervención en redes sociales en trabajo social comunitario. En primer lugar, los profesionales del trabajo social han subrayado la 
Completar recursos

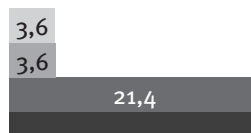

Examinar redes

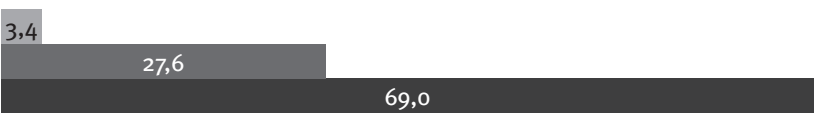

Contribuir desarrollo

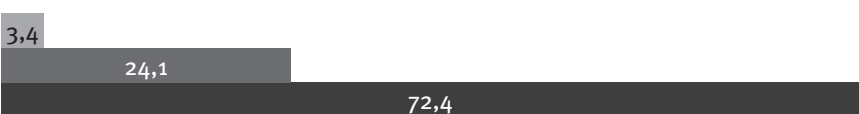

Identificar tejido relaciona

Activar recursos resilencia

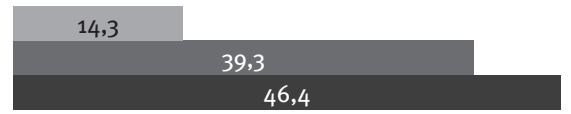

\section{6,9}

Mediar personas y sistemas
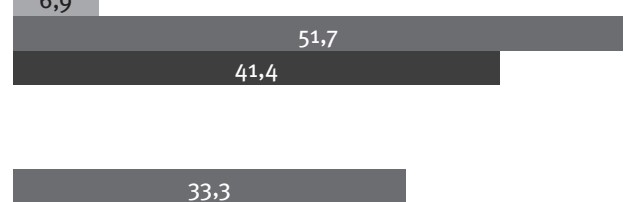

66,7

Aumentar autocuidado

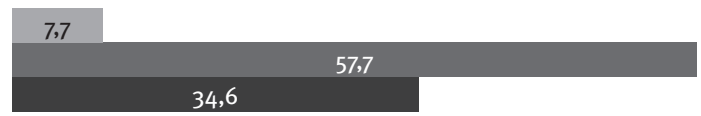

6,9

Mediar interacciones

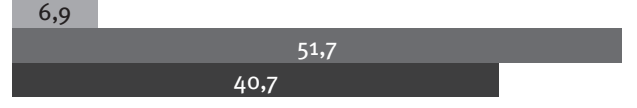

\section{7,4}

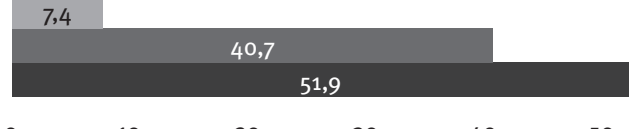

60

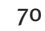

80

Nada importante

Poco importante

Bastante importante

Muy importante

Fuente: Rodríguez (2014b).

Gráfico 9. Técnicas específicas de intervención desde la red social (\%)

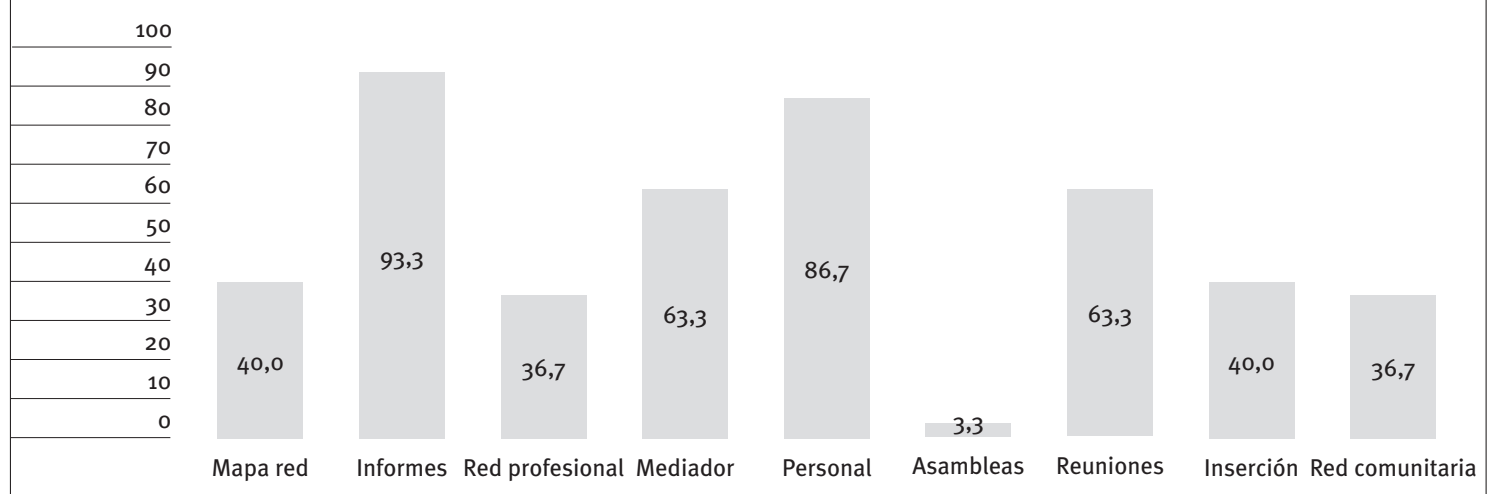

Fuente: Rodríguez (2014b). 
importancia de los valores y los principios éticos, al haberlos considerado la base fundamental de su práctica profesional y, también que "los procesos, las tareas y las prácticas para el empowerment de las comunidades les van a permitir asumir la responsabilidad colectiva de su propio desarrollo"' (Kenny, 2006: 10). En este sentido, "la práctica del empowerment necesita un desarrollo desde los niveles social y político, y también desde las dimensiones personales de los sujetos, cuya práctica implica, a su vez, inserción social, participación y justicia social” (Rodríguez, 2014a: 38).

El trabajo social tiene una base instrumental formada por un conjunto de referencias teórico-metodológicas, a partir de las cuales los trabajadores sociales han considerado de intervención prioritaria en redes sociales a aquellos individuos que se encuentran en una situación de dificultad social sin red de apoyo, o red muy escasa, y en situación de aislamiento físico y social, coincidiendo ambas respuestas con los problemas sociales comunitarios que han sido señalados por los profesionales; y a su vez, responden, desde la identificación de la red social, a la importancia de conocer la existencia de personas de la comunidad que las ayudan. Los cambios permanentes en la situación social y económica nos llevan a la necesidad de optimizar las redes existentes, y en este sentido, "el objetivo del trabajador social es, por una parte, insertar a las personas aisladas en las redes de solidaridad y, por otra, reforzar las redes de solidaridad existentes y, eventualmente, participar en la creación de nuevas redes" (De Robertis y Pascal, 2007: 328). La mayoría de los trabajadores sociales participantes en la investigación han identificado los referentes teórico-metodológicos del modelo sistémico y la intervención en redes sociales como soportes teóricos principales de su intervención, al igual que los roles de 'facilitador de procesos individuales y colectivos', 'experto consultor' y 'referente organizativo y de recursos', siendo sus funciones principales 'identificar el tejido relacional' y 'conocer los recursos existentes en la comunidad', habiendo subrayado también la utilización de técnicas de carácter más individual y menos colectivo, lo que nos refleja que la intervención que se realiza es de tipo individual, frente a otra de carácter más global. A este respecto, compartimos con Villalba que "lo que se precisa para orientar las intervenciones son herramientas conceptuales que apunten a examinar las fortalezas" (2011: 287).

A modo de conclusión, podemos afirmar, a la luz de los resultados obtenidos, que el trabajo social comunitario en el ámbito local necesita enfoques basados en las fortalezas para trabajar con las comunidades, donde el énfasis se ponga en la construcción y el fortalecimiento de las redes sociales, para que se movilicen sus recursos hacia un cambio positivo. "El propósito del desarrollo de la comunidad es apoyar y dar forma a la creación de redes formales e informales con el objetivo de facilitar y hacer real el empowerment de la acción colectiva" (Gilchrist, 2009: 95). Porque, tal como afirman Ennis y West (2010), las relaciones son lo que crean una comunidad y, por tanto, son la piedra angular de su desarrollo. Así, el municipio "tendría que asumir mayores responsabilidades, convirtiéndose en una entidad mediadora de todas, o de buena parte, de las acciones realizadas para luchar contra la pobreza y la exclusión social” (Ruivo, 2000: 16). 
ABALLÉA, F.; RIDDER, G.; y GADÉA, C. (2003): “Processos em fase de reconhecimento e concorrências profissionais", en CHOPART, J. N. (ed.), Os novos desafios do trabalho social: dinâmicas de um campo profissional, Oporto, Porto Editora, págs. 203-221.

ADAMS, R. (2008): Empowerment, Participation and Social Work, $3^{\underline{a}}$ ed., Nueva York, Palgrave Macmillan.

ARNAUT-BRAVO, S. (2010): "La lucha contra la pobreza en el origen del Trabajo Social”, en GUTIÉRREZ, A. (ed.), Orígenes y desarrollo del Trabajo Social, Madrid, Ediciones Académicas, págs. 25-53.

BÁEZ, J.; y PÉREZ DE TUDELA, J. (2007): Investigación cualitativa, Madrid, ESIC.

BAUMAN, Z. (2011): Cuarenta y cuatro cartas desde el mundo líquido, Barcelona, Paidós.

BONET, J. (2006): “La vulnerabilidad relacional. Análisis del fenómeno y pautas de intervención", Redes. Revista Hispana para el Análisis de Redes Sociales, vol. 11, no 4 , págs. 1-17 [rhttp:// revista-redes.rediris.es/pdf-vol11/Vol11_4.pdf)]

CARDOSO, J. F. (2012): “Acção Social nos Municípios portugueses, potencialidades e limitações" [tesis doctoral], Lisboa, Instituto Universitário de Lisboa, Departamento de Ciência Política e Políticas Públicas.

CEA, Ma A. (1996): Metodología cuantitativa. Estrategias y técnicas de investigación social, Madrid, Síntesis.

CHOPART, J. N. (2003): Os novos desafios do trabalho social: dinâmicas de um campo profissional, Oporto, Porto Editora.

COLEMAN, J. (1988): "Social capital and the creation of human capital”, American Journal of Sociology, vol. 94 , págs. 95-120.
DE ROBERTIS, C. (2000): "Respondiendo a las nuevas situaciones desde los fundamentos del trabajo social", en VV.AA., Trabajo Social. Compromiso y equilibrio. IX Congreso Estatal de Diplomados en Trabajo Social y Asistentes Sociales, Santiago de Compostela, Colexio Oficial de Diplomados en Traballo Social de Galicia, págs. 15-35.

DE ROBERTIS, C.; y PASCAL, H. (2007): La intervención colectiva en trabajo social. La acción con grupos y comunidades, Buenos Aires, Hvmanitas.

DEL PINO, M. D. (2000): “La dimensión individual y colectiva del trabajo social en los servicios sociales comunitarios", en MARTÍNEZ, $M^{-}$- J. (coord.), Para el trabajo social: aportaciones teóricas y prácticas, Granada, Maristán, págs. 275-300.

DOMINELLI, L. (2009): “Repositing social work”, en ADAMS, R.; DOMINELLI, L.; y PAYNE, M., Social Work. Themes, Issues and Critical Debates, $3^{\mathrm{a}}$ ed., Basingstoke, Macmillan, págs. 13-25.

ENNIS, G.; y WEST, D. (2010): “Exploring the potential of social network analysis in asset-based community development practice and research", Australian Social Work, vol. 63, nํㅜ 4, págs. 404-417.

FERRARIO, F. (2009): Il lavoro di rete nel servizio sociale. Gli operatori fra solidarietà e istituzioni, $5^{\underline{a}}$ ed., Roma, Carocci.

FERREIRA, J. M. L. (2011): Serviço social e Modelos de Bemestar para a Infância. 'Modus operandi' do Assistente Social na Promoçao da Protecção à Criança e à Familia, Lisboa, Ouid Juris.

FOLGHERAITER, F. (2012): Teorìa e metodología del Servizio Sociale. La prospettiva de rete, $4^{\underline{a}}$ ed., Milán, Franco Agnelli. 
- (2011): Fondamenti di metologia relazionale. La lógica sociales dell'aiuto, $2^{\underline{a}}$ ed., Trento, Erickson.

FRANS, D. J. (1993): “A scale for measuring social worker empowerment”, Social Work Practice, vol. 3 , nํㅜ 3, págs. 312-318.

GIDDENS, A. (2007): Europa en la era global, Barcelona, Paidós Ibérica.

GILCHRIST, A. (2009): The Well-Connected Community: A Networking Approach to Community Development, $2^{\underline{a}}$ ed., Bristol, Policy Press.

GOETZ, J.; y LECOMPTE, M. (1988): Etnografía y diseño cualitativo en investigación educativa, Madrid, Morata.

GUBA, E.; y LINCOLN, Y. (1982): "Epistemological and methodological bases of naturalistic inquiry", Educational Communication \& Technology Journal, vol. 30, nํㅜ 4, págs. 233-253.

KENNY, S. (2006): Developing Communities for the Future, $3^{\underline{a}}$ ed., Melbourne, Cengage Learning.

LEE, J. A. B. (2001): The Empowerment Approach to Social Work Practice, $2^{\mathrm{a}}$ ed., Nueva York, Columbia University Press.

MARCHIONI, M (coord.) (2001): Comunidad y cambio social. Teoría y praxis de la acción comunitaria, Madrid, Popular.

MARSHALL, M. T. (2006): "Sujeto social y desarrollo comunitario", en EROLES, C. (coord.), Familia(s), estallido, puente y diversidad: una mirada transdisciplinaria de los derechos humanos, Buenos Aires, Espacio.

PÉREZ DÍAZ, V. (2008): El malestar de la democracia, Barcelona, Crítica.

PUTNAM, R. (ed.) (2003): El declive del capital social. Un estudio internacional sobre las sociedades y el sentido comunitario, Barcelona, Galaxia Gutenberg; Barcelona, Círculo de Lectores.

REAMER, F. G. (2013): "Social work in a digital age: Ethical and risk management challenges", Social Work, vol. 58, n- 2 [<http://www2.sunysuffolk.edu/ bybeem/SS22/SW\%20in\%20a\%2odigital\%20 age.pdf>, consultado el 20-4-14].

RODRÍGUEZ, M. D. (2014a): “El enfoque del empowerment: piedra angular del Trabajo Social Comunitario en el siglo XXI", Trabajo Social Hoy, nํㅜㄱ, págs. 27-42.
- (2014b): "Redes sociales: un nuevo cuadro metodológico de intervención en Trabajo Social Comunitario" [tesis doctoral], Madrid, Universidad Complutense de Madrid, Departamento de Trabajo Social y Servicios Sociales.

- (2012): "Ciudadanía, comunidad, municipio y redes sociales. La fuerza de lo imprevisto", Revista Intervenção Social, nํ40, págs. 125-142 [<http://revistas.lis.ulusiada.pt/index.php/is/ article/view/1212>].

RUIVO, F. (2000): Poder Local e Exclusão Social, Coimbra, Quarteto.

RUIZ, J. I.; e ISPIZUA, Mํㅡㄹ A. (1989): La descodificación de la vida cotidiana. Métodos de investigación cualitativa, Bilbao, Universidad de Deusto.

SEGAL, E. A.; GERDES, K. E.; y STEINER, S. (2013): An Introduction to the Profession of Social Work. Becoming a Change Agent, Belmont, Brooks/ Cole Cengage Learning.

SONG, L. (2011): "The extent and correlates of the utilization of empowerment strategies: A survey of social workers in the field of partner violence", British Journal of Social Work, nํ 41, págs. 1.016-1.037.

SUBIRATS, J. (dir.) (2007): Los servicios sociales de atención primaria ante el cambio social, Madrid, Ministerio de Trabajo y Seguridad Social.

UNWIN, P.; y HOGG, R. (2012): Effective Social Work with Children and Families: A Skills Handbook, Londres, Sage Publications.

VILLALBA, C. (2011): “El concepto de resiliencia en trabajo social”, en CORDERO MARTÍN, G.; CORDERO RAMOS, N.; y FERNÁNDEZ MARTÍN, M. I., El mosaico de la intervención social. Métodos y conceptos en trabajo social, Sevilla, Aconcagua Libros, págs. 275-293.

- (2009): "Del contacto en el escenario social a la construcción de redes sociales y fuentes de apoyo social”, XI Congreso Estatal de Trabajo Social, Zaragoza.

- (1995): “Intervención en redes”, Documentación Social, nํ 98, págs. 105-119.

VISCARRET, J. J. (2007): Modelos y métodos de intervención en Trabajo Social, Madrid, Alianza. 